\title{
Involvement of Genetic Factors in Multiple Sclerosis
}

\author{
Laura Ferrè ${ }^{1,2,3,4}$, Massimo Filippi ${ }^{1,2,4,5,6}$ and Federica Esposito ${ }^{1,2,3 *}$ \\ ${ }^{1}$ Neurology Unit, Istituto di Ricovero e Cura a Carattere Scientifico San Raffaele Scientific Institute, Milan, Italy, \\ ${ }^{2}$ Neurorehabilitation Unit, Istituto di Ricovero e Cura a Carattere Scientifico San Raffaele Scientific Institute, Milan, Italy, \\ ${ }^{3}$ Laboratory of Human Genetics of Neurological Disorders, Institute of Experimental Neurology, Division of Neuroscience, \\ Istituto di Ricovero e Cura a Carattere Scientifico San Raffaele Scientific Institute, Milan, Italy, ${ }^{4}$ Vita-Salute San Raffaele \\ University, Milan, Italy, ${ }^{5}$ Neurophysiology Unit, Istituto di Ricovero e Cura a Carattere Scientifico San Raffaele Scientific \\ Institute, Milan, Italy, ${ }^{6}$ Neuroimaging Research Unit, Institute of Experimental Neurology, Division of Neuroscience, Istituto di \\ Ricovero e Cura a Carattere Scientifico San Raffaele Scientific Institute, Milan, Italy
}

Keywords: multiple sclerosis, genetic factors, inflammation, neurodegeneration, disease severity, treatment response

\section{INTRODUCTION}

Multiple sclerosis (MS) is the most common chronic inflammatory disease of the central nervous system (CNS) that affects young adults between 20 and 40 years of age, with a higher prevalence in females (female-to-male ratio 3:1) (Dilokthornsakul et al., 2016). Even though its etiology is still debated, it has historically been considered an immune-mediated disease, whose histopathological hallmarks are inflammation, demyelination, and neurodegeneration (Frohman et al., 2006; Lassmann, 2018). Indeed, acute demyelinating lesions characterized by blood brain barrier breakdown, lymphocyte infiltration, oligodendrocyte loss and astrocytic activation, mainly located in myelin-rich white-matter areas, are distinctive of the disease. Nonetheless, in more

OPEN ACCESS

Edited by:

Bert A. 'T Hart,

University Medical Center

Groningen, Netherlands

Reviewed by:

Giovanni Ristori,

Sapienza University of Rome, Italy

*Correspondence:

Federica Esposito esposito.federica@hsr.it

Specialty section:

This article was submitted to Cellular Neuropathology,

a section of the journal

Frontiers in Cellular Neuroscience

Received: 01 October 2020 Accepted: 09 November 2020 Published: 01 December 2020

Citation:

Ferrè L, Filippi $M$ and Esposito $F$ (2020) Involvement of Genetic Factors

in Multiple Sclerosis.

Front. Cell. Neurosci. 14:612953.

doi: 10.3389/fncel.2020.612953 recent years it became clear that axonal loss and primary demyelination in the absence of acute inflammatory infiltrates are also present since the earliest stages of the disease (Henderson et al., 2009), challenging the idea that neurodegeneration is secondary to CNS inflammation (Louapre and Lubetzki, 2015). As a matter of fact, it is still unclear whether neurodegeneration is a direct consequence of inflammatory CNS injury or whether it represents a primitive independent process, and a better understanding of the interplay between these two aspects of the disease is warranted. In this perspective, studies investigating genetic factors associated with MS onset and progression can help to shed light on this matter; in fact, compared to cross-sectional histopathological studies that do not permit to establish temporal and/or causal relationship, genetic association studies allow to infer causality and to pinpoint molecular pathways and cell types that play a key role in MS pathogenesis.

\section{HLA ASSOCIATION}

The first MS-associated genetic risk locus discovered in 1972 was located in the human leukocyte antigen (HLA) class I region on chromosome 6 (Jersild et al., 1972; Naito et al., 1972). Since then, several HLA class I and II alleles have been associated with an increased risk of developing MS (Hauser et al., 1989; Patsopoulos et al., 2013) or were found to be protective (Fogdell-Hahn et al., 2000).

The strongest association has been demonstrated with the HLA DRB1*1501 allele (Hauser et al., 1989; Oksenberg et al., 2004; Patsopoulos et al., 2013) that confers an almost 3-fold increased risk of MS. The most recent genome-wide association study (GWAS) in MS (Patsopoulos et al., 2019) identified up to 32 independent MS risk-variants in the major histocompatibility complex (MHC) region and, overall, the MHC locus alone is estimated to explain 20\% of MS heritability (Patsopoulos et al., 2019). 
The HLA locus maps on chromosome $6 \mathrm{p} 21.3$ and encompasses more than 200 genes, with important roles in maturation and regulation of the $\mathrm{T}$ cell compartment as well as in other immunological processes, supporting the hypothesis that MS is an immune-mediated disease arising from a dysregulation of the peripheral immune system which targets the CNS. Consistently, a fine-mapping study of the MHC region (Patsopoulos et al., 2013) has shown that some of the MS-associated variants in this locus affect the amino-acidic sequence of the peptide-binding groove, potentially influencing antigen recognition and $\mathrm{T}$-cell repertoire specificity.

\section{GENOME-WIDE ASSOCIATION STUDIES OF MS SUSCEPTIBILITY}

Since 1970s, the knowledge of MS genetic architecture has extraordinarily advanced and several GWAS have been performed, identifying hundreds of additional MS genetic risk variants outside the MHC locus (The International Multiple Sclerosis Genetics Consortium et al., 2007; Bahlo et al., 2009; Baranzini et al., 2009; Sanna et al., 2010; Patsopoulos et al., 2011, 2019; Sawcer et al., 2011; Beecham et al., 2013). GWAS are particularly helpful in the discovery of novel disease variants, because they do not depend on "a priori" assumption on disease pathogenesis and can help to unravel novel molecular mechanisms underlying the disease.

The first MS GWAS (The International Multiple Sclerosis Genetics Consortium et al., 2007) was performed on 931 family trios and identified variants in the interleukin-7 receptor (IL7R) and in the interleukin-2 receptor (IL2RA) loci (Gregory et al., 2007; Weber et al., 2008), again pointing to the crucial role played by the immune system. In the following years, the collaborative efforts of the International MS Genetic Consortium (IMSGC) allowed to combine MS cohorts from several countries, improving the statistical power and leading to a remarkable increase in the number of the identified MS risk variants. In 2011, the analysis of 9,772 MS cases and 17,376 controls from 15 different countries, followed by a replication step in 4,218 cases and 7,296 healthy controls (Sawcer et al., 2011), allowed to confirm most of the previously reported associations and to detect 29 novel risk variants. Noteworthy, the prioritized effects were enriched in genes involved in lymphocyte functions and in particular in $\mathrm{T}$ cell activation and proliferation. Moreover, genes linked to Vitamin D metabolism, such as CYP27B1, CYP24A1, and genes coding for targets of MS immunemodulatory therapies, including VCAM1 for natalizumab and IL2RA for daclizumab, were also highlighted. More recently, the greatest advancement in MS genetics field was made possible by a meta-analysis involving 47,351 MS subjects and 68,284 healthy controls (Patsopoulos et al., 2019) that increased the number of MS-associated variants up to 200 autosomal SNPs outside the $\mathrm{MHC}$ region and one variant on the $\mathrm{X}$ chromosome. Once again, the identified variants seem to be enriched in loci which are active in immune cells, both from the adaptive ( $\mathrm{T}$ and $\mathrm{B}$ cells) and the innate (natural killer and dendritic cells) immune compartments; conversely there was no enrichment of
MS susceptibility loci in CNS tissues and, also when analyzing induced pluripotent stem cell derived neurons and purified human astrocytes or microglia, the only significant enrichment was detected in microglial cells, suggesting that also CNS resident immune compartment could play a role in the disease. Similarly, when assessing the effect of the selected variants on gene expression modulation in specific tissues (eQTL effect) and when evaluating the biological pathways enriched in MS genes, findings were indicative of the central role of immune cells, while putative functional consequences in neurons and other CNS cells need further investigations. A more recent study (Factor et al., 2020) applied a so-called "outside variant approach" to identify additional risk variants that physically interact with MS-associated genes and to pinpoint cell-types in which these genes exert their pathogenic role. As expected, the majority of MS loci were predicted to act in T cells, but also $B$ cells and myeloid cells were involved, underlining the role of both the innate and adaptive immune system. Moreover, the authors also identified 6 loci that were predicted to act in the CNS and they hypothesized that a dysregulation of transcriptional elongation in oligodendrocytes could contribute to MS pathogenesis.

Finally, the main role played by the immune system is also reflected in the significant proportion of MS risk variants that is shared with other autoimmune diseases (Richard-Miceli and Criswell, 2012), such as type 1 diabetes, rheumatoid arthritis and intestinal bowel diseases, even though sometimes with opposite effect. The common background with other immunological conditions seems to imply the existence of a genetic basis predisposing to peripheral immune dysregulation; other risk factors, such as environmental exposures and/or infections, could then play a role in determining the target tissue and phenotypic expression of this autoimmune predisposition.

\section{THE ROLE OF LOW-FREQUENCY/RARE VARIANTS}

Classic GWAS take advantage of very large numbers of enrolled subjects to screen hundreds of thousands common variants over the entire genome but are largely underpowered to detect association with low-frequency and rare variants. More recently, some studies investigated the contribution of rare variants in MS heritability using a candidate gene approach or a whole exome analysis in sporadic and familiar MS cases. Specifically, a study conducted in about 68,000 subjects identified four novel variants driving MS risk independently of common-variant signals, which are implicated in regulatory $\mathrm{T}$ cell homeostasis and regulation, IFN gamma biology, and NFkB signaling (Mitrovič et al., 2018). Another study identified an increased burden of rare variants with high predicted pathogenicity in MS cases compared to controls when analyzing genes of the inflammasome pathway (e.g., NLRP1/3, CASP1) (Vidmar et al., 2019). Similarly, an additional work on 34 multi-incident MS families also identified some putative risk variants implicating the inflammasome pathway (NLRP12), as well as other biological processes involved 
in innate immunological responses, again stressing the role of immune factors in the disease (Vilariño-Güell et al., 2019).

\section{GENETIC FACTORS INFLUENCING DISEASE SEVERITY}

Overall, genetic studies on MS susceptibility seem to denote a major role for immune-related processes in disease pathogenesis; however, these studies do not take into consideration the highly variable degree of neurodegeneration and disability accumulation that characterize the disease. For this reason, genetic studies investigating variants associated with disease severity represent a complementary approach in understanding MS pathological basis.

First of all, candidate gene studies have assessed the role of genes implicated in MS susceptibility in determining disease course, failing to identify any significant association (Jensen et al., 2010) except for the HLA-DRB1*1501 allele (Hauser et al., 2000; Barcellos et al., 2003). Interestingly enough, a subsequent GWAS (Baranzini et al., 2009) also failed to identify an enrichment of associated genes implicated in immunological functions. On the contrary, when disease severity was assessed using MRI measures such as brain volume and T2 lesion load, there was an over-representation of genes related to neural processes (e.g., GRIN2A, NLGN1) such as glutamate signaling and axon guidance. Similarly, a network analysis on results from a GWAS that used brain glutamate concentrations as endophenotype (Baranzini et al., 2010) identified a module enriched in genes implicated in glutamate biology that correlated with the level of brain atrophy and $\mathrm{N}$-Acetylaspartate decline over time, pointing to a possible role of excitotoxicity in modulating disease severity.

Furthermore, an additional GWAS (Briggs et al., 2011) that investigated variants associated with disease severity using the MS severity score (MSSS) and in which a pathway analysis was applied showed an enrichment of genes involved in axon guidance and signaling and neuronal processes as well as with interferon $\alpha / \beta$ receptor binding and antigen processing and presentation.

\section{PHARMACOGENETIC STUDIES}

Finally, also works investigating genetic determinants of response to disease modifying treatments can add to the knowledge of the biological basis of the disease.

Interferon $\beta$ (IFN $\beta$ ) was the first drug to be approved for MS and is also the most investigated in pharmacogenomic studies; specifically, the first of these studies focused on genes known to be implicated in its mechanism of action applying a candidate gene approach. At least 15 genes associated with IFN $\beta$ response were identified (Cunningham et al., 2005; Martínez et al., 2006; Gross et al., 2011; Kulakova et al., 2012), although not all the associations have been validated in independent cohorts. Not surprisingly, associated polymorphisms mapped to region encoding type I IFN receptor (Cunningham et al., 2005), IFN response-element sequences, IFN regulatory transcription factors (Gross et al., 2011) and to loci encoding different cytokine genes (IL10, IFN $\gamma$ ) (Martínez et al., 2006; Kulakova et al., 2012). Candidate studies also evaluated the role of the HLA class II locus, and mainly of the HLA DR2 haplotype, in influencing IFN $\beta$ response, but no significant association was found (Gross et al., 2011).

More interesting were the results of GWAS on response to IFN $\beta$ (Byun et al., 2008; Comabella et al., 2009; Esposito et al., 2015; Clarelli et al., 2017; Mahurkar et al., 2017); these investigations not only confirmed the role of genes implicated in the IFN $\beta$ pathway, such as IFNAR2, but also identified genes involved in neuronal functions like GPC5 (Byun et al., 2008), the glutamate receptors GRIA3 (Comabella et al., 2009), GRM3 and GRIK2 (Clarelli et al., 2017) and SLC9A9 (Esposito et al., 2015), which has been linked to neuronal excitability (Gu et al., 2001), pointing to a possible role for glutamate metabolism and excitotoxicity in modulating drug response. Similarly, candidate gene studies on response to glatiramer acetate (GA) (Fusco et al., 2001; Grossman et al., 2007; Tsareva et al., 2012; Kulakova et al., 2017) focused mainly on immunerelated genes and found that the clinical outcome during GA treatment is associated with HLA class II genes (Fusco et al., 2001; Gross et al., 2011) and other genes involved in $\mathrm{T}$ cell activation (TCRB, Grossman et al., 2007), antigen presentation (CD86) or proinflammatory signaling [(IL1R1 and IL12RB2, Grossman et al., 2007), (CCR5, Tsareva et al., 2012)]. Additionally, a recent GWAS on more than 2,500 GAtreated MS patients (Ross et al., 2017) selected a set of 4 SNPs mapping to HLA-DQB2, MBP, UVRAG, and ZAK, which distinguished signature-positive or negative patients, signaturepositive subjects displaying a better outcome over signaturenegative individuals.

\section{DISCUSSION}

The results of genetic studies on MS susceptibility (The International Multiple Sclerosis Genetics Consortium et al., 2007; Patsopoulos et al., 2019) identified a strong enrichment of genes and biological processes implicated in immune functions, suggesting that a dysregulation of immune responses, promoted by the genetic background and potentially triggered by environmental factors, is the main mechanism of the disease. Overall, apart from a single study that predicted $6 \mathrm{MS}$ risk loci to be mainly active in CNS cells, where they can disrupt oligodendrocyte maturation (Factor et al., 2020), no particular enrichment in genes and pathways involved in CNS development and regulation was found, implying that neurodegeneration is more likely to derive from an inflammatory insult than to be a primary process itself. However, these studies mainly enrolled patients with a relapsing-remitting form of the disease, which is characterized by a high level of inflammatory activity. Thus, it is possible that genetic variants affecting the degree of neuronal damage and disability accumulation were not adequately pickedup by such studies. Indeed, genetic studies that focused on disease severity showed genes involved in neuronal processes, and in particular in glutamate biology, as associated with the degree of disability progression and neurodegeneration (Baranzini et al., 
2009, 2010). Noteworthy, some of these genes and pathways were also associated with response to MS treatments (Comabella et al., 2009; Esposito et al., 2015; Clarelli et al., 2017). Unfortunately, most of the genetic studies on MS severity and treatment response have been performed in relatively modest datasets and lack a validation in larger, independent cohorts due to the difficulty in obtaining detailed longitudinal clinical information in huge cohorts. Nonetheless, these results seem to suggest that inflammation is not the only determinant of disease course and also neuronal factors can modulate the level of neurodegeneration and disability progression in MS. Further

\section{REFERENCES}

Bahlo, M., Booth, D. R., Simon, A., Broadley, B., Foote, S. J., Griffiths, L. R., et al. (2009). Genome-wide association study identifies new multiple sclerosis susceptibility loci on chromosomes 12 and 20. Nat. Genet. 41, 824-828. doi: $10.1038 /$ ng.396

Baranzini, S. E., Srinivasan, R., Khankhanian, P., Okuda, D. T., Nelson, S. J., Matthews, P. M., et al. (2010). Genetic variation influences glutamate concentrations in brains of patients with multiple sclerosis. Brain 133, 2603-2611. doi: 10.1093/brain/awq192

Baranzini, S. E., Wang, J., Gibson, R. A., Galwey, N., Naegelin, Y., Barkhof, F., et al. (2009). Genome-wide association analysis of susceptibility and clinical phenotype in multiple sclerosis. Hum. Mol. Genet. 18, 767-778. doi: $10.1093 / \mathrm{hmg} / \mathrm{ddn} 388$

Barcellos, L. F., Oksenberg, J. R., Begovich, A. B., Martin, E. R., Schmidt, S., Vittinghoff, E., et al. (2003). HLA-DR2 dose effect on susceptibility to multiple sclerosis and influence on disease course. Am. J. Hum. Genet. 72, 710-716. doi: $10.1086 / 367781$

Beecham, A. H., Patsopoulos, N. A., Xifara, D. K., Davis, M. F., Kemppinen, A., Cotsapas, C., et al. (2013). Analysis of immune-related loci identifies 48 new susceptibility variants for multiple sclerosis. Nat. Genet. 45, 1353-1360. doi: $10.1038 /$ ng. 2770

Briggs, F. B. S., Shao, X., Goldstein, B. A., Oksenberg, J. R., Barcellos, L. F., and De Jager, P. L. (2011). Genome-wide association study of severity in multiple sclerosis. Genes Immun. 12, 615-625. doi: 10.1038/gene. 2011.34

Byun, E., Caillier, S. J., Montalban, X., Villoslada, P., Fernández, O., Brassat, D., et al. (2008). Genome-wide pharmacogenomic analysis of the response to interferon beta therapy in multiple sclerosis. Arch. Neurol. 65, 337-344. doi: 10.1001/archneurol.2008.47

Clarelli, F., Liberatore, G., Sorosina, M., Osiceanu, A. M., Esposito, F., Mascia, E., et al. (2017). Pharmacogenetic study of long-term response to interferon- $\beta$ treatment in multiple sclerosis. Pharmacogenomics J. 17, 84-91. doi: $10.1038 /$ tpj. 2015.85

Comabella, M., Craig, D. W., Morcillo-Suárez, C., Río, J., Navarro, A., Fernández, M., et al. (2009). Genome-wide scan of 500000 singlenucleotide polymorphisms among responders and nonresponders to interferon beta therapy in multiple sclerosis. Arch. Neurol. 66, 972-978. doi: 10.1001/archneurol.2009.150

Cunningham, S., Graham, C., Hutchinson, M., Droogan, A., O’Rourke, K., Patterson, C., et al. (2005). Pharmacogenomics of responsiveness to interferon IFN-beta treatment in multiple sclerosis: a genetic screen of 100 type I interferon-inducible genes. Clin. Pharmacol. Ther. 78, 635-646. doi: 10.1016/j.clpt.2005.08.018

Dilokthornsakul, P., Valuck, R. J., Nair, K. V., Corboy, J. R., Allen, R. R., and Campbell, J. D. (2016). Multiple sclerosis prevalence in the United States commercially insured population. Neurology 86, 1014-1021. doi: 10.1212/WNL.0000000000002469

Esposito, F., Sorosina, M., Ottoboni, L., Lim, E. T., Replogle, J. M., Raj, T., et al. (2015). A pharmacogenetic study implicates SLC9a9 in multiple sclerosis disease activity. Ann. Neurol. 78, 115-127. doi: 10.1002/ana.24429 studies on larger datasets are required to validate these results and to help identifying potential therapeutic targets to halt neurodegeneration in MS.

\section{AUTHOR CONTRIBUTIONS}

LF: design and conceptualization of the study and manuscript writing. MF: revision of the manuscript for intellectual content. FE: conceptualization of the study and revision of the manuscript for intellectual content. All authors contributed to the article and approved the submitted version.

Factor, D. C., Barbeau, A. M., Allan, K. C., Hu, L. R., Madhavan, M., Hoang, A. T., et al. (2020). Cell type-specific intralocus interactions reveal oligodendrocyte mechanisms in MS. Cell 181, 382-395.e21. doi: 10.1016/j.cell.2020.03.002

Fogdell-Hahn, A., Ligers, A., Grønning, M., Hillert, J., and Olerup, O. (2000). Multiple sclerosis: a modifying influence of HLA class I genes in an HLA class II associated autoimmune disease. Tissue Antigens 55, 140-148. doi: 10.1034/j.1399-0039.2000.550205.x

Frohman, E. M., Racke, M. K., and Raine, C. S. (2006). Multiple sclerosisthe plaque and its pathogenesis. N. Engl. J. Med. 354, 942-955. doi: 10.1056/NEJMra052130

Fusco, C., Andreone, V., Coppola, G., Luongo, V., Guerini, F., Pace, E., et al. (2001). HLA-DRB1*1501 and response to copolymer-1 therapy in relapsing-remitting multiple sclerosis. Neurology 57, 1976-1979. doi: 10.1212/WNL.57.11.1976

Gregory, S. G., Schmidt, S., Seth, P., Oksenberg, J. R., Hart, J., Prokop, A., et al. (2007). Interleukin 7 receptor $\alpha$ chain (IL7R) shows allelic and functional association with multiple sclerosis. Nat. Genet. 39, 1083-1091. doi: $10.1038 / \mathrm{ng} 2103$

Gross, R., Healy, B. C., Cepok, S., Chitnis, T., Khoury, S. J., Hemmer, B., et al. (2011). Population structure and HLA DRB1 1501 in the response of subjects with multiple sclerosis to first-line treatments. J. Neuroimmunol. 233, 168-174. doi: 10.1016/j.jneuroim.2010.10.038

Grossman, I., Avidan, N., Singer, C., Goldstaub, D., Hayardeny, L., Eyal, E., et al. (2007). Pharmacogenetics of glatiramer acetate therapy for multiple sclerosis reveals drug-response markers. Pharmacogenet. Genomics 17, 657-666. doi: 10.1097/FPC.0b013e3281299169

Gu, X. Q., Yao, H., and Haddad, G. G. (2001). Increased neuronal excitability and seizures in the $\mathrm{Na}+/ \mathrm{H}+$ exchanger null mutant mouse. Am. J. Physiol. Cell Physiol. 281, C496-C503. doi: 10.1152/ajpcell.2001.281.2.C496

Hauser, S. L., Fleischnick, E., Weiner, H. L., Marcus, D., Awdeh, Z., Yunis, E. J., et al. (1989). Extended major histocompatibility complex haplotypes in patients with multiple sclerosis. Neurology 39, 275-277. doi: 10.1212/WNL.39.2.275

Hauser, S. L., Oksenberg, J. R., Lincoln, R., Garovoy, J., Beck, R. W., Cole, S. R., et al. (2000). Interaction between HLA-DR2 and abnormal brain MRI in optic neuritis and early MS. Neurology 54, 1859-1861. doi: 10.1212/WNL.54.9.1859

Henderson, A. P. D., Barnett, M. H., Parratt, J. D. E., and Prineas, J. W. (2009). Multiple sclerosis: distribution of inflammatory cells in newly forming lesions. Ann. Neurol. 66, 739-753. doi: 10.1002/ana.21800

Jensen, C. J., Stankovich, J., Van der Walt, A., Bahlo, M., Taylor, B. V., van der Mei, I. A. F., et al. (2010). Multiple sclerosis susceptibility-associated SNPs do not influence disease severity measures in a cohort of Australian MS patients. PLoS ONE 5:e10003. doi: 10.1371/journal.pone.0010003

Jersild, C., Svejgaard, A., and Fog, T. (1972). HL-A antigens and multiple sclerosis. Lancet 1, 1240-1241. doi: 10.1016/S0140-6736(72)90962-2

Kulakova, O., Bashinskaya, V., Kiselev, I., Baulina, N., Tsareva, E., Nikolaev, R., et al. (2017). Pharmacogenetics of glatiramer acetate therapy for multiple sclerosis: the impact of genome-wide association studies identified disease risk loci. Pharmacogenomics 18, 1563-1574. doi: 10.2217/pgs-2017-0058

Kulakova, O. G., Tsareva, E. Y., Boyko, A. N., Shchur, S. G., Gusev, E. I., Lvovs, D., et al. (2012). Allelic combinations of immune-response genes as possible composite markers of IFN- $\beta$ efficacy in multiple sclerosis patients. Pharmacogenomics 13, 1689-1700. doi: 10.2217/pgs.12.161 
Lassmann, H. (2018). Multiple sclerosis pathology. Cold Spring Harb. Perspect. Med. 8:a028936. doi: 10.1101/cshperspect.a028936

Louapre, C., and Lubetzki, C. (2015). Neurodegeneration in multiple sclerosis is a process separate from inflammation: Yes. Mult. Scler. 21, 1626-1628. doi: $10.1177 / 1352458515587598$

Mahurkar, S., Moldovan, M., Suppiah, V., Sorosina, M., Clarelli, F., Liberatore, G., et al. (2017). Response to interferon-beta treatment in multiple sclerosis patients: a genome-wide association study. Pharmacogenomics J. 17, 312-318. doi: $10.1038 /$ tpj.2016.20

Martínez, A., de las Heras, V., Mas Fontao, A., Bartolom,é, M., de la Concha, E. G., Urcelay, E., et al. (2006). An IFNG polymorphism is associated with interferon-beta response in Spanish MS patients. J. Neuroimmunol. 173, 196-199. doi: 10.1016/j.jneuroim.2005.12.002

Mitrovič, M., Patsopoulos, N. A., Beecham, A. H., Dankowski, T., Goris, A., Dubois, B., et al. (2018). Low-frequency and rare-coding variation contributes to multiple sclerosis risk. Cell 175, 1679-1687.e7. doi: 10.1016/j.cell.2018.0 9.049

Naito, S., Namerow, N., Mickey, M. R., and Terasaki, P. I. (1972). Multiple sclerosis: association with HL-A3. Tissue Antigens 2, 1-4.

Oksenberg, J. R., Barcellos, L. F., Cree, B. A. C., Baranzini, S. E., Bugawan, T. L., Khan, O., et al. (2004). Mapping multiple sclerosis susceptibility to the HLA-DR locus in African Americans. Am. J. Hum. Genet. 74, 160-167. doi: 10.1086/380997

Patsopoulos, N. A., Baranzini, S. E., Santaniello, A., Shoostari, P., Cotsapas, C., Wong, G., et al. (2019). Multiple sclerosis genomic map implicates peripheral immune cells and microglia in susceptibility. Science 365:eaav7188. doi: $10.1126 /$ science.aav7188

Patsopoulos, N. A., Barcellos, L. F., Hintzen, R. Q., Schaefer, C., van Duijn, C. M., Noble, J. A., et al. (2013). Fine-mapping the genetic association of the major histocompatibility complex in multiple sclerosis: HLA and non-HLA effects. PLoS Genet. 9:e1003926. doi: 10.1371/journal.pgen.100 3926

Patsopoulos, N. A., Esposito, F., Reischl, J., Lehr, S., Bauer, D., Heubach, J., et al. (2011). Genome-wide meta-analysis identifies novel multiple sclerosis susceptibility loci. Ann. Neurol. 70, 897-912. doi: 10.1002/ana. 22609

Richard-Miceli, C., and Criswell, L. A. (2012). Emerging patterns of genetic overlap across autoimmune disorders. Genome Med. 4:6. doi: 10.1186/gm305

Ross, C. J., Towfic, F., Shankar, J., Laifenfeld, D., Thoma, M., Davis, M., et al. (2017). A pharmacogenetic signature of high response to copaxone in late-phase clinical-trial cohorts of multiple sclerosis. Genome Med. 9:50. doi: 10.1186/s13073-017$0436-y$

Sanna, S., Pitzalis, M., Zoledziewska, M., Zara, I., Sidore, C., Murru, R., et al. (2010). Variants within the immunoregulatory CBLB gene are associated with multiple sclerosis. Nat. Genet. 42, 495-497. doi: 10.1038/ng.584
Sawcer, S., Hellenthal, G., Pirinen, M., Spencer, C. C. A., Patsopoulos, N. A., Moutsianas, L., et al. (2011). Genetic risk and a primary role for cellmediated immune mechanisms in multiple sclerosis. Nature 476, 214-219. doi: 10.1038/nature10251

The International Multiple Sclerosis Genetics Consortium, Hafler, D. A., Compston, A., Sawcer, S., Lander, E. S., Daly, M. J., et al. (2007). Risk alleles for multiple sclerosis identified by a genomewide study. N. Engl. J. Med. 357, 851-862. doi: 10.1056/NEJMoa073493

Tsareva, E. Y., Kulakova, O. G., Boyko, A. N., Shchur, S. G., Lvovs, D., Favorov, A. V., et al. (2012). Allelic combinations of immune-response genes associated with glatiramer acetate treatment response in Russian multiple sclerosis patients. Pharmacogenomics 13, 43-53. doi: 10.2217/pgs.11.136

Vidmar, L., Maver, A., Drulović, J., Sepčić, J., Novaković, I., Ristič, S., et al. (2019). Multiple sclerosis patients carry an increased burden of exceedingly rare genetic variants in the inflammasome regulatory genes. Sci. Rep. 9:9171. doi: 10.1038/s41598-019-45598-x

Vilariño-Güell, C., Zimprich, A., Martinelli-Boneschi, F., Herculano, B., Wang, Z., Matesanz, F., et al. (2019). Exome sequencing in multiple sclerosis families identifies 12 candidate genes and nominates biological pathways for the genesis of disease. PLoS Genet. 15:e1008180. doi: 10.1371/journal.pgen.1008180

Weber, F., Fontaine, B., Cournu-Rebeix, I., Kroner, A., Knop, M., Lutz, S., et al. (2008). IL2RA and IL7RA genes confer susceptibility for multiple sclerosis in two independent European populations. Genes Immun. 9, 259-263. doi: 10.1038 /gene.2008.14

Conflict of Interest: MF is Editor-in-Chief of the Journal of Neurology; received compensation for consulting services and/or speaking activities from Bayer, Biogen Idec, Merck-Serono, Novartis, Roche, Sanofi Genzyme, Takeda, and Teva Pharmaceutical Industries; and receives research support from Biogen Idec, Merck-Serono, Novartis, Roche, Teva Pharmaceutical Industries, Italian Ministry of Health, Fondazione Italiana Sclerosi Multipla, and ARiSLA (Fondazione Italiana di Ricerca per la SLA). FE has received compensation for consulting services and/or speaking activities from Novartis, Sanofi Genzyme, Almirall, TEVA and Merck-Serono.

The remaining author declares that the research was conducted in the absence of any commercial or financial relationships that could be construed as a potential conflict of interest.

Copyright (C) 2020 Ferrè, Filippi and Esposito. This is an open-access article distributed under the terms of the Creative Commons Attribution License (CC BY). The use, distribution or reproduction in other forums is permitted, provided the original author(s) and the copyright owner(s) are credited and that the original publication in this journal is cited, in accordance with accepted academic practice. No use, distribution or reproduction is permitted which does not comply with these terms. 